\title{
François Hubault (coord.), Risques psychosociaux : quelle réalité, quels enjeux pour le travail?
}

Toulouse, Octarès éditions, collection « Le travail en débats ", série «Séminaire Paris 1 », 2011

\section{Christophe Massot}

\section{(2) OpenEdition}

\section{Journals}

Édition électronique

URL : http://journals.openedition.org/travailemploi/5919

DOI : 10.4000/travailemploi.5919

ISSN : 1775-416X

Éditeur

DARES - Ministère du Travail

Édition imprimée

Date de publication : 15 mars 2013

Pagination : 66-67

ISSN : 0224-4365

\section{Référence électronique}

Christophe Massot, « François Hubault (coord.), Risques psychosociaux : quelle réalité, quels enjeux pour le travail ? », Travail et Emploi [En ligne], 133 | janvier-mars 2013, mis en ligne le 01 janvier 2013, consulté le 22 septembre 2020. URL : http://journals.openedition.org/travailemploi/5919 ; DOI : https://doi.org/10.4000/travailemploi.5919

Ce document a été généré automatiquement le 22 septembre 2020.

(c) Direction de l'animation de la recherche, des études et des statistiques (Dares) 


\section{François Hubault (coord.), Risques psychosociaux: quelle réalité, quels enjeux pour le travail?}

Toulouse, Octarès éditions, collection « Le travail en débats », série « Séminaire Paris 1 », 2011

\section{Christophe Massot}

\section{RÉFÉRENCE}

François Hubault (coord.), Risques psychosociaux : quelle réalité, quels enjeux pour le travail ?, Toulouse, Octarès éditions, collection « Le travail en débats ", série « Séminaire Paris $1 », 2011,195$ p.

1 L'ouvrage collectif, coordonné par François Hubault, rassemble treize communications du séminaire de Paris 1 qui s'est déroulé les 8,9 et 10 juin 2009 sur le thème : « Risques psychosociaux (RPS) : quelle réalité, quels enjeux pour le travail ?».

2 François Hubault, dans l'introduction de l'ouvrage, pose deux questions absolument essentielles pour qui s'intéresse aux relations entre santé et travail. La première est celle de l'opposition entre rupture et continuité: quelle réelle nouveauté se tient derrière l'apparition récente mais imposante de l'expression "risques psychosociaux » (RPS) ? Sommes-nous en présence d'une nouvelle appellation d'un phénomène déjà existant ou bien d'un nouveau phénomène sans précédent? La seconde, liée à la première, est celle de la distinction entre l'effet et la cause: si nous sommes en présence d'une rupture, concerne-t-elle les effets ou les causes des RPS ? Sommes-nous confrontés à la transformation des effets d'un mal ancien aux causes connues, ou bien à l'avènement de nouvelles causes d'un mal jusqu'ici inconnu?

Les communications, toutes tendues par ce double questionnement, sont l'œuvre de consultants ou d'intervenants en entreprise, essentiellement d'ergonomes (Bourgeois, Coutarel, Cru, De Gasparo, Hubault, Lapeyrière, Le Port, Morlet), mais aussi de 
chercheurs exerçant dans des domaines aussi divers que la sociologie (Evette), la statistique (Cristofari), l'économie (du Tertre, Ughetto), l'histoire (Hatzfeld), la philosophie (Barksat, Benattar) et l'architecture (Fenker). La richesse de l'ouvrage provient certes de la diversité des auteurs, mais aussi du fait que tous abordent ces questions d'un point de vue théorique et pratique, soutenant la même ambition de ne pas séparer l'action et la compréhension.

D'abord, l'ambiguïté de l'appellation même de RPS - qui tend à construire le problème dans le schéma classique de l'employeur «exposant» le salarié, et le contraint juridiquement à l'en protéger (Ughetto) - ne peut dissimuler cette réalité : l'acteur au travail ne se réduit pas à un objet exposé au risque et à la souffrance. Le risque n'est pas une réalité en soi venant affecter l'activité de l'extérieur. À ce titre, différentes contributions nous montrent comment l'acteur, l'activité et la situation entretiennent des relations de réciprocité, d'adaptation et de reconfiguration réciproque (Cru et Lapeyre, Ughetto). L'activité n'est pas un objet inerte placé dans une situation. Ensuite, forts de ce constat, les auteurs, avec des termes différents, se rejoignent pour qualifier les RPS de "pathologie de l'engagement» (p. 188). La souffrance, qui sous-tend l'expression RPS, se déploie depuis «l'écart entre le geste intentionné et le geste réalisé, la part du geste contrariée, empêchée, privée » (Bourgeois, De Gasparo, p. 90). Il ne s'agit pas seulement d'un déséquilibre mais d'un « empêchement d'accomplir et de s'accomplir [précipité] dans le corps » (p. 188). C'est ici que les RPS échappent à la métrologie ou à la causalité car ce qui n'a pas été fait, ce qui ne sera pas fait, ce qui aurait pu et dû être fait ne peut être mesuré, évalué ou normé. Les auteurs se placent dans la continuité des travaux initiés par la clinique de l'activité, notamment de sa réflexion en termes de « pouvoir d'agir » ${ }^{1}$.

Enfin Hubault, dans sa stimulante conclusion, prolonge la réflexion : si les RPS ont une cause, au-delà de la transformation de l'environnement de travail, au-delà même de la restriction du pouvoir d'agir des salariés au travail, elle se situerait dans « une crise du rapport au réel » (p. 192). Mais le réel n'est, pas plus que l'activité, défini comme une substance avec laquelle l'individu perdrait contact. Le réel se déploie comme un rapport entre mondes hétéronomes et l'activité « expérimente un rapport au réel » (p. 189). Hubault propose quatre registres que l'activité a en charge de tenir et d'accorder : la santé, la subjectivité, l'entreprise et la société. Ainsi parler de crise du rapport au réel, c'est dire que l'activité n'a plus les moyens d'expérimenter une mise en lien de registres hétéronomes. Aux deux questions initiales, il est répondu, sur un versant théorique, que les RPS forment l'écho, spécifiquement contemporain, de « l'empêchement de l'activité à lier » (p. 192).

6 L'autre dimension importante de cet ouvrage collectif est la recherche, par les praticiens, d'une mise en œuvre de ces orientations dans le travail de prévention. Comment s'y prendre, s'interrogent Damien Cru et Solange Lapeyrière, deux ergonomes, "pour ne pas constituer le risque psychosocial [...] comme des choses en soi » (p. 140) dans le travail de prévention? Quelles pratiques donner à la prévention s'il n'est pas possible de construire un système d'événements externes puis de prévenir le risque de sa réalisation?

7 Ces deux ergonomes refusent en premier lieu la situation « confortable " consistant à rédiger, suite à une demande standard, un diagnostic, partagé ou non. Ils disent chercher à «tracer le chemin en avançant et avancer sur un fil » (p. 149). Mais de quel fil s'agit-il ? Pour Fabrice Bourgeois et Sandro De Gasparo, reprenant les savoirs acquis 
par la prévention des troubles musculo-squelettiques (TMS), il faut d'abord retrouver l'écart entre l'intention et l'acte réalisé, cet empêchement du geste. Une fois les " composantes altérées du geste identifiées ", l'enjeu est alors "de définir les moyens de leur restauration, leur réactivation, leur revalorisation dans l'intelligence pratique, dans les coopérations, dans l'investissement subjectif» (p. 87). Différents exposés précis donnent à voir ce travail spécifique, encore en formation, de prévention. Le travail de prévention ne consiste pas à découvrir l'événement, l'environnement, l'acte constituant la cause extérieure du trouble mais à retrouver le «développement des savoir-faire de prudence et les conditions permettant d'anticiper la survenue des situations de débordement»(p. 96). La prévention est d'abord une investigation tournée vers l'activité comme capacité à créer des liens, des compromis entre des mondes hétérogènes, à constituer des savoir-faire de prudence, puis une promotion du pouvoir d'agir. Comme Socrate avec la maïeutique, le praticien mobilise moins un savoir préconstitué qu'il ne redécouvre une réalité déjà présente. Mais dès lors, la responsabilité de l'expertise sur la situation de travail est fondamentalement renversée : l'expertise de la situation pathogène de travail est nécessairement détenue in fine par celui qui tient l'activité.

8 Ce renversement de responsabilité inscrit les RPS dans une dimension politique. Si les RPS se répandent, c'est que les acteurs au travail n'ont plus la capacité et le pouvoir de creuser le rapport au réel, de constituer des savoir-faire de prudence, de déployer leur pouvoir d'agir. "L'explosion des troubles psychosociaux témoigne selon nous des ravages du glissement de la subordination vers la soumission et l'assujettissement. [...] Dès lors, la prévention des risques psychosociaux sonne comme une convocation, scientifique tout autant que politique, professionnelle [et] morale» (p. 194), écrit François Hubault dans les derniers paragraphes de l'ouvrage. Si Nicolas Hatzfeld retrace le processus social et politique d'émergence des TMS, ce travail est encore à prolonger pour les RPS ${ }^{2}$. La question que semble ouvrir cet ouvrage, avec d'autres, est celle de la constitution des RPS comme enjeu social et politique: comment la problématisation et la prévention des RPS reformulent la question du fondement et de la répartition des pouvoirs dans les organisations?

\section{NOTES}

1. Clot Y. (2008), Travail et pouvoir d'agir, Paris, Presses universitaires de France, collection « Le travail humain ».

2. Ce travail a en partie été effectué depuis par le même auteur dans une livraison récente de la revue: cf. Nicolas Hatzfeld (2012), «Les risques psychosociaux: quelles correspondances anciennes aux débats récents? " in "Les risques psychosociaux au travail : d'une "question de société" à des questions scientifiques », Travail et emploi, n 129, pp. 11-22. 


\section{AUTEURS}

\section{CHRISTOPHE MASSOT}

Chercheur associé au Laboratoire d'Économie et de Sociologie du Travail (LEST) 